\title{
TRATAMENTO DA ESQUISTOSSOMOSE MANSÔNICA NA CRIANÇA COM OXAMNIQUINE SOB FORMA DE XAROPE ${ }^{\ddagger}$
}

\author{
Helena Moura * Helena Nisquier** Sonia Bechara ** Ana \\ Lúcia C. Domingues *** Amaury Coutinho ****
}

\begin{abstract}
São apresentados os resu/tados preliminares do tratamento da esquistossomose mansônica com oxamniquine, sob forma de xarope, em 40 crianças, com idade variando de 5 a 14 anos. 18 pertenciam ao sexo masculino e 22 ao feminino. A dose utilizada foi de $20 \mathrm{mg} / \mathrm{k} \mathrm{em}$ uma única aplicação - 25 pacientes (Grupo A) ou em 2 tomadas com intervalo de 12 hs - 15 pacientes (Grupo B). A tolerância foi em geral boa, sobretudo no Grupo $B$ de pacientes, onde $73,3 \%$ não apresentou qualquer sintoma colateral, em comparação com $44 \%$ do Grupo A. Os sintomas mais freqüentes foram tontura $(22,5 \%)$ e sonolência $(20 \%)$. 5 pacientès do Grupo $A$ apresentaram distúrbio de conduta sob forma de excitação psíquica ou agressividade passageiras. A cura parasitológica lcomprovação entre o 49 e o $60^{\circ}$ mês pós-tratamentol foi observada em $70 \%$ dos pacientes tratados $190 \%$ no Grupo $A$ e $50 \%$ no Grupo B). O baixo índice, nesse último Grupo, possive/mente deveu-se ao longo intervalo entre as 2 tomadas (12hs). Houve grande redução do número de ovos eliminados nos pacientes não curados.
\end{abstract}

Em trabalhos anteriores, Coutinho e Domingues $^{1,2,3}$ apresentaram sua experiência favorável com a Oxamniquine, injetável e oral (cápsulas) no tratamento da esquistossomose mansônica em adultos. Em continuação, a presente investigação teve por finalidade o estudo da mesma droga, sob apresentação de xarope, em crianças acometidas de esquistossomose mansô. nica. A pesquisa visou particularmente a eficácia e a tolerância da nova forma do medicamento, especial para crianças, sob duas diferentes posologias: $20 \mathrm{mg} / \mathrm{kg}$ em dose única no período de jejum, e $20 \mathrm{mg} / \mathrm{kg}$ dividida em duas tomadas, com intervalo de 12 horas.

\# Suporte parcial do Conselho Nacional de Desenvolvimento Cientifico e Tecnológico CNPq - Plano integrado de Pesquisa sobre Esquistossomose.

* Prof. Adjunto de Clínica Pediátrica da U.F.Pe.

* Residentes de Clínica Pediatrica.

** Bolsista do CNPq

* * Prof. Titular de Clínica Médica da U.F.Pe. Recebido para publicação em 26.10.1976. 
TABELA III

Relação dos doentes tratados. Esquema $A(20 \mathrm{mg} / \mathrm{k}$ dose única)

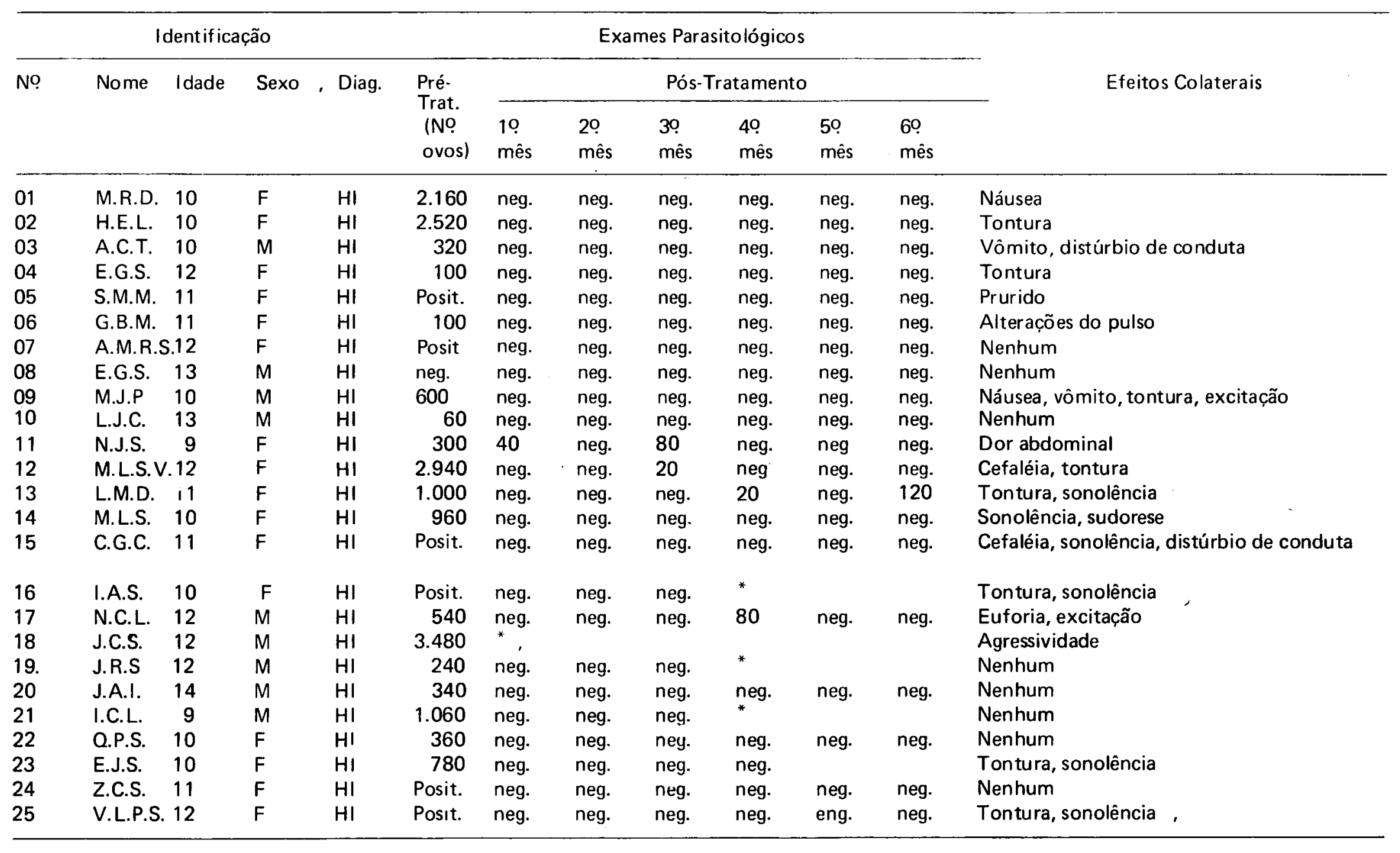

*Paciente não retornou para controle , 


\section{MATERIAL (PACIENTES) E MÉTODOS}

Foram estudadas 40 crianças, internadas na Clínica Pediátrica do Hospital Infantil de Pernambuco, IMIP, divididas em do is grupos:

Grupo A - 25 pacientes - $20 \mathrm{mg} / \mathrm{kg}$ D.U.
Grupo B - 25 pacientes - 200mg/kg (10mg/ kg b.i.d.)

Os dados gerais sobre os pacientes estão contidos na Tabela I e a distribuição etária na Tabela 11 , onde se verifica predominância na faixa dos 10 aos 13 anos.

TABELA I

Tratamento da Esquistossomose em crianças com Oxamnique-Xarope Dados Gerais

\begin{tabular}{|c|c|c|c|c|c|}
\hline \multirow{2}{*}{$\begin{array}{l}\text { No Total } \\
\text { pacientes }\end{array}$} & \multirow[t]{2}{*}{ Idade } & \multirow[t]{2}{*}{ Sexo } & \multirow{2}{*}{$\begin{array}{l}\text { Forma } \\
\text { Clinica }\end{array}$} & \multicolumn{2}{|c|}{ Grupos } \\
\hline & & & & Dose & NO casos \\
\hline \multirow[t]{2}{*}{40} & $\begin{array}{c}5 \text { a } 14 \\
\text { anos }\end{array}$ & $\begin{array}{l}M-18 \\
F-22\end{array}$ & $\begin{array}{l}H I-38 \\
H E-2\end{array}$ & $\begin{array}{l}\text { A-20 mg } \times 1 \\
\text { B-10 mg } \times 2\end{array}$ & $\begin{array}{l}25 \\
15\end{array}$ \\
\hline & (Média 10.5) & & & & \\
\hline
\end{tabular}

$\mathrm{HI}$ - Hepato intestinal - HE - Hepato esplênico

TABELA II

Distribuição dos pacientes pela idade

\begin{tabular}{cccc}
\hline Idade & Grupo A & Grupo B & Total \\
\hline$<9$ & 0 & $5^{*}$ & $5^{*}$ \\
9 & 2 & 0 & 2 \\
10 & 8 & 2 & 10 \\
11 & 6 & 3 & 9 \\
12 & 6 & 1 & 7 \\
13 & 2 & 4 & 1 \\
14 & 1 & 0 & 40 \\
\hline Total & 25 & 15 & 10.5 \\
\hline Média & 11.0 & 10.0 & 15 \\
\hline
\end{tabular}

* Dois pacientes tinham 5 anos, $1-6$ anos, 1-7 anos e 1-8 anos

A fim de ser estudada a tolerância do medicamento e a possibilidade de algum efeito tóxi. $\infty$ para o lado do rim, fígado, sangue, coração e sistema nervoso foram realizados, em todos os pacientes, uma ficha clínica de acompanhamento e os seguintes exames complementares: exame de urina, dosagem de uréia e creatinina no sangue, hemograma, transaminases e fosfatase alcalina, todos eles antes, $48 \mathrm{~h}$ e 10 dias após o quimioterápico; ECG, realizado antes de $24 \mathrm{~h}$ depois e EEG, antes, às 12 e $24 \mathrm{~h}$ após o tratamento.
O estudo da eficácia foi analisado através de exame de fezes pela técnica de Hoffman e da contagem de ovos de S. mansoni pela técnica de Kato.

Os exames laboratoriais foram efetuados no Laboratório de análise do IMIP e no Laboratório de Pesquisa Clínica do Departamento de Medicina Clínica (Disciplina de Gastroenterologia e Hepatologia). Os eletrocardiogramas foram realizados e interpretados pela Dra. Fernan. da Andrade e os eletroencefalogramas pelo Dr. 
José Bezerra, aos quais agradecemos a valiosa cooperação.

\section{RESULTADOS}

\section{Efeitos colaterais}

Os efeitos colaterais são observados, de forma discriminada, nas Tabelas III e IV e de forma resumida na Tabela $V$. Verifica-se que houve nítida predominância de todos os paraefeitos no Grupo A de dose única, (56\%) em relação ao Grupo B de dose fracionada. $(26,6 \%)$ Como nos estudos anteriores, a tontura (leve a moderada) foi a queixa mais freqüente $(22.5 \%$ no total de pacientes). Segue-se sonolência (20.0\%) e outros sintomas da esfera neuropsíquica: cefaléia (moderada a intensa) em $7.5 \%$, alterações de conduta (agressividade e agitação) em 3 casos (7.5\%) e excitação com excesso de euforia (brincadeira constante dentro do hospital) em 2 pacientes $(5.0 \%)$. Todos esses sintomas, todavia, foram passageiros; a tontura, sonolência e cefaléia durando poucas horas e os outros sintomas de 1 a 2 dias.

TABELA IV

RELAÇAO DOS DOENTES TRATADOS' ESQUEMA B $(20 \mathrm{mg} / \mathrm{k}$ EM 2 DOSES C/INTERVALOS DE 12HS)

\begin{tabular}{|c|c|c|c|c|c|c|c|c|c|c|c|c|}
\hline \multirow[b]{3}{*}{ N. } & \multicolumn{4}{|c|}{ IDENTIFICAÇAOO } & \multicolumn{7}{|c|}{ EXAMES PARASITOLOGICOS } & \multirow{3}{*}{ EFEITOS COLATERAIS } \\
\hline & \multirow[b]{2}{*}{ NOME } & \multirow[b]{2}{*}{ IDADE } & \multirow[b]{2}{*}{ SEXO } & \multirow[b]{2}{*}{ DIAG. } & \multirow{2}{*}{$\begin{array}{l}\text { PRÉ- } \\
\text { TRAT. } \\
\text { (n'o ovos) }\end{array}$} & \multicolumn{6}{|c|}{ PÓS-TRATAMENTO } & \\
\hline & & & & & & $\begin{array}{l}10 \\
\text { mês }\end{array}$ & $\begin{array}{l}2^{\circ} \\
\text { mès }\end{array}$ & $\begin{array}{l}3^{\circ} \\
\text { mês }\end{array}$ & $\begin{array}{l}4 \stackrel{0}{ } \\
\text { mês }\end{array}$ & $\begin{array}{l}5{ }^{\circ} \\
\text { mès }\end{array}$ & $\begin{array}{l}6 !^{\circ} \\
\text { mês }\end{array}$ & \\
\hline 26 & O.F.S. & 5 & $\mathbf{F}$ & $\mathrm{HI}$ & 60 & neg. & neg. & neg. & $*$ & & & Náusea \\
\hline 27 & A.A.S & 11 & $M$ & $\mathrm{HE}$ & 1.020 & 400 & neg. & neg. & 440 & 80 & 160 & Nenhum \\
\hline 28 & A.L.S. & 13 & $M$ & $\mathrm{HI}$ & 760 & neg. & neg. & neg. & neg. & neg. & 80 & Nenhum \\
\hline 29 & R.M.S. & 5 & $M$ & $\mathrm{HI}$ & 420 & neg. & neg. & neg. & neg. & neg. & neg. & Tontura \\
\hline 30 & W.M.S. & 7 & $M$ & $\mathrm{HI}$ & 920 & neg. & neg. & neg. & neg. & 100 & neg. & Nenhum \\
\hline 31 & E.M.S. & 10 & $F$ & $\mathrm{HI}$ & 1.800 & 20 & neg. & neg. & 20 & neg. & neg. & Dor muscalar \\
\hline 32 & E.A.S. & 10 & $M$ & $\mathrm{HI}$ & 2.200 & neg. & neg. & neg. & neg. & neg. & neg. & Nenhum \\
\hline 33 & W.M.F. & 8 & $\mathbf{F}$ & HI & 1.360 & neg. & neg. & neg. & neg. & & & Nenhum \\
\hline 34 & J.M.O. & 13 & $M$ & $\mathrm{HI}$ & 660 & neg. & neg. & neg. & neg. & 40 & neg. & Dor abdominal, tontura, anorexia, febre \\
\hline 35 & E.G.S. & 6 & $F$ & HI & 2.340 & neg. & neg. & neg. & neg. & 80 & neg. & Sonolência \\
\hline 36 & R.A.D. & 13 & M & HE & 1.100 & neg. & & & & & & Dor abdominal \\
\hline 37 & T.M.A. & 13 & $\mathbf{F}$ & HI & 500 & neg. & neg. & $*$ & & & & Nenhum \\
\hline 38 & E.G.S. & 11 & $\mathbf{F}$ & HI & 1.780 & neg. & neg. & neg. & neg. & neg. & neg. & Nenhum \\
\hline 39 & J.R.S. & 12 & $M$ & $\mathrm{HI}$ & 1.200 & neg. & neg. & & & & & Nenhum \\
\hline 40 & J.M.S. & 11 & $M$ & HI & 520 & neg. & neg. & neg. & neg. & neg. & neg. & Nenhum \\
\hline
\end{tabular}

*Paciente não retomou para controle

TABELA V

Oxamniquine-Xarope em crianças - Efeitos Colaterais

\begin{tabular}{lccrr}
\hline \multicolumn{1}{c}{ Sintomas } & $\begin{array}{c}\text { Grupo A } \\
(20 \mathrm{mg} \times 1)\end{array}$ & $\begin{array}{c}\text { Grupo B } \\
(10 \mathrm{mg} \times 2)\end{array}$ & \multicolumn{2}{c}{ Total } \\
\hline No Casos & 25 & 15 & 40 & - \\
\hline Nenhum sintoma & $11(44 . \%)$ & $11(73.3 \%)$ & 22 & 55.0 \\
Tontura & 8 & 1 & 9 & 22.5 \\
Sonolência & 6 & 2 & 8 & 20.0 \\
Alteração de conduta & 3 & 0 & 3 & 7.5 \\
(agressividade) & 2 & 0 & 2 & 5.0 \\
Excitação-Euforia & 2 & 1 & 3 & 7.5 \\
Cefaléia & 2 & 1 & 3 & 7.5 \\
Nauséa & 2 & 0 & 2 & 5.0 \\
Vômitos & 0 & 1 & 1 & 2.5 \\
Febre & & & & \\
\hline
\end{tabular}


Dada a sua importância, transcrevemos abaixo um resumo de cada uma das 5 observações clínicas de alterações do comportamento ou excitação e na tabela VI a súmula dos referidos casos. Além da constatação de que os sintomas neuro-psiquicos só aparecem no grupo de pacientes que recebem dose única da droga 120 $\mathrm{mg} / \mathrm{k}$ ) verificou-se a presença, em alguns casos, na fase prévia ao tratamento, de possíveis condições predisponentes.

\section{TABELA VI \\ OXAMNIQUINE-XAROPE EM CRIANÇAS \\ Alterações neuro-ssíquicas}

\begin{tabular}{llllll}
\hline $\begin{array}{l}\text { Iniciais } \\
\text { NN., }\end{array}$ & $\begin{array}{l}\text { Idade } \\
\text { (anos) }\end{array}$ & Sexo & $\begin{array}{l}\text { Sintoma } \\
\text { neuro-psiq. }\end{array}$ & $\begin{array}{l}\text { Outros } \\
\text { sintomas }\end{array}$ & $\begin{array}{l}\text { Condições } \\
\text { pré-trat. }\end{array}$ \\
\hline $\begin{array}{c}\text { A.C.T. } \\
3\end{array}$ & 10 & $\mathrm{M}$ & $\begin{array}{l}\text { alt. conduta } \\
(10 \mathrm{~d})\end{array}$ & $\begin{array}{l}\text { vômito } \\
1 \text { vez } \\
\text { cefaléia }+\end{array}$ & "nervosismo" normal \\
& & & & &
\end{tabular}

\begin{tabular}{|c|c|c|c|c|c|}
\hline $\begin{array}{l}\text { M.J.P. } \\
9\end{array}$ & 10 & $M$ & $\begin{array}{l}\text { excitação, } \\
\text { euforia } \\
\left(10^{\circ} \text { e } 2{ }^{\circ} \text { d.) }\right.\end{array}$ & $\begin{array}{l}\text { nausea }+++ \text { alt. conduta } \\
\text { vômito } 4 \text { vezes } \\
\text { tontura ++ }\end{array}$ & $\begin{array}{l}\text { "ondas lentas } \\
\text { agudas" as } 12 \mathrm{hs}\end{array}$ \\
\hline
\end{tabular}

\begin{tabular}{|c|c|c|c|c|c|c|}
\hline $\begin{array}{l}\text { C.G.C. } \\
15\end{array}$ & 11 & $F$ & $\begin{array}{l}\text { alt. conduta } \\
\text { (1. d.) }\end{array}$ & $\begin{array}{l}\text { tontura ++ } \\
\text { sonolência }+\end{array}$ & $\begin{array}{l}\text { "nervosismo, } \\
\text { timidez" }\end{array}$ & $\begin{array}{l}\text { modificação } \\
\text { p/melhor (? }\end{array}$ \\
\hline $\begin{array}{l}\text { N.C.L } \\
17\end{array}$ & 12 & $M$ & $\begin{array}{l}\text { excitação, } \\
\text { euforia (1? } \\
\text { d) }\end{array}$ & - & $\begin{array}{l}\text { retardo } \\
\text { mental }\end{array}$ & $\begin{array}{l}\text { exarcebação } \\
\text { irritativo às }\end{array}$ \\
\hline J.C.S. & 12 & $M$ & $\begin{array}{l}\text { alt. conduta } \\
\left(10^{\circ} \text { e } 2^{\circ} \mathrm{d} .\right)\end{array}$ & - & $\begin{array}{l}\text { crises } \\
\text { convulsivas } \\
\text { (controladas } \\
\text { c/comital) }\end{array}$ & normal (?) \\
\hline
\end{tabular}

Por outro lado, em 3 das referidas crianças constatou-se discreta modificação do EEG bastante precoce, isto é, 12 hs após a terapêutica, retornando à situação pré-tratamento no exame de 24 hs. Essas modificações, no entanto, não tiveram caráter de especificidade e foram diferentes entre si: Um caso ( $n$. 9) apresentou discreta desorganização da atividade de base"; outro (n: 17) revelou "exacerbação de foco irritativo temporal posterior esquerdo", e finalmente o terceiro (n. 15) apresentou, ao inverso, "melhor organização da atividade de base, em relação ao traçado anterior que mostrava excesso de atividade lentas, provalmente relacionadas a imaturidade".

É interessante registrar que o caso $n$. 18, que apresentava no pré-tratamento um quadro de "Grande Mal", controlado por Comital, não apresentou crises convulsivas, após a droga, mas apenas uma alteração de comportamento sob forma de agressividade e o EEG não se modificou essencialmente; apenas no exame das 12 hs apareceram, durante o sono espon. taneo, "focos irritativos muito ativos nas regiões rolandicas relacionadas a patologia anterior", os quais, no entanto, não foram evidenciados no exame pré-tratamento, por não ter se obtido sono espontâneo.

Resumo das 5 observaç̃̃es:

I - N. 3, A.C.T. masc. 10 anos, forma HI.

Após receber o medicamento $(20 \mathrm{mg} / \mathrm{kg}$-dose única, em jejum) manifesta, pouco tempo depois, intensa agitação, inadaptação ao ambiente e insubordinação às ordens do pessoal médico e para-médico. Apesar da observação constante exercida sobre ele, foge do Hospital, sendo encontrado nas proximidades logo mais. Redobrada a vigilância, procura fugir 
novamente, sem êxito. No dia seguinte está mais calmo, tendo-se conseguido que permanecesse no Hospital 48 hs para exame de controle. Fora isso, queixa-se de discreta cefaléia e vômito uma vez. $O$ EEG, antes do tratamento e os obtidos 12 e 24 hs após nada revelam de anormal.

II - N. 9, M.J.P, masc., 10 anos, forma $\mathrm{HI}$.

Apresentava, antes da terapia específica, distúrbio de conduta caracterizado por constante desobedienncia aos pais e permanência fora de casa, pelo que se encontrava em tratamento. No Hospital, no dia que recebeu a droga $(20 \mathrm{mg} / \mathrm{k} \quad$ D.U. $)$ é dificil a sua adaptação ao meio ambiente, por se apresentar excitado e muito eufórico. brincando com todo mundo e utilizando todo material das enfermarias nas brincadeiras sem atender as advertências do pessoal médico e para-médico. Essas manifestacões duram todo dia e continuam, menos acentuadas, no dia seguinte, e são acompanhadas, no $1^{\mathrm{O}}$ dia, de forte nausea, 4 vezes, moderada tontura e sonolência. Dois EEG, feitos antes não apresentaram anormalidade; todavia, 12 hs após receber a medicação verifica-se "discreta desorganização da atividade de base com alterações paroxisticas por ondas lentas e ondas agudas de projeção difusa e bilateral" e, 24 horas depois do tratamento, constata-se o retorno ao aspecto anormal anterior.

$1 I I$ - N. 15, C.G.C, fem. 11 anos, forma HII.

Segundo a genitora, a criança era tímida e muito nervosa. Após o recebimento da droga $(20 \mathrm{mg} / \mathrm{k} \mathrm{D.U.)}$ torna-se irreverente com os superiores e agressiva com as outras crianças. A noite, é impossível mantê-la na Enfermaria, tendo sido solicitado à família levá-la para a residência, com o compromisso de trazê-la, no dia seguinte para os exames e observações, no que fomos atendidos. Nesse dia, volta ao Hospital mais calma e acessivel, al permanecendo. Vale acrescentar que um irmão desta criança falecera 3 dias antes de seu internamento. Os outros sintomas foram: cefaléia, sonolência e tontura. O EEG prévio revela "excesso de atividades lentas sem alterações paroxisticas Irelacio- nado provavelmente a imaturidade"; 12 hs após a medicação, observa-se "em relação ao anterior melhor organização da atividade de base" e 24 hs depois "aspecto semelhante ao pré-tratamento, com excesso de freqüèncias lentas"

IV - No 17, N.C.L. masc. 12 anos, forma HI.

O paciente apresentava retardo mental, bastante inferior a idade cronológica. 6 meses antes havia referência a um quadro discreto de insuficiência cardíaca congestiva. No dia do tratamento $120 \mathrm{mg} / \mathrm{k}$ D.U.) mostrava-se excitado e com alegria incontida parecendo muito feliz. O EEG prévio apresenta "foco irritativo temporal posterior esquerdo". 12 hs após o medicamento", há exarcebação do referido foco, com alterações bem mais freqüentes", enquanto com $24 \mathrm{hs,}$ a intensidade das alterações volta ao ritimo pré-tratamento.

$V$ - N. 18 , J.C.S. masc. 12 anos, forma HI.

O paciente era portador de "Grande Mal" desde os 4 anos, estando em tratamento, em nosso próprio serviço e perfeitamente controlado com o uso de Comital. No dia do tratamento $(20 \mathrm{mg} / \mathrm{k}$ D.U. $)$ fica bastante agressivo com as crianças da Enfermaria e com o pessoal para-médico, não obedecendo as normas do Hospital. O EEG prévio, feito em vigilia e com ativação pela hiperpnéia, nada revela de anormal. $O$ de 12 hs mostra o mesmo resultado, porém, durante o sono expontâneo são revelados "focos irritativos muito ativos nas regiões rolandicas, relacionados a patologia anterior". Nas 24 hs, o traçado é idêntico ao pré-tratamento, não se tendo obtido sono expontàneo.

\section{Função hepática}

Em relação as transaminases, não foram observadas alterações significativas após a quimioterapia (Tabela VII), mesmo naqueles pacientes que já apresentavam discretas elevações pré-tratamento (Tabela VIII). O mesmo se pode dizer em referência à fosfatase alcalina.

TABELA VII

OXAMNIQUINE-XAROPE, EM CRIANÇAS

Valores médios das transaminases

TGO

\begin{tabular}{lcccc}
\hline & N: ${ }^{\circ}$ casos & O hora & 48 horas & $10^{\circ}$ dia \\
\cline { 2 - 5 } Grupo A & 25 & 33.8 & 34.5 & 30.3 \\
Grupo B & 14 & 37.7 & 34.0 & 32.0 \\
Total & 39 & 35.2 & 34.4 & 31.1 \\
\hline
\end{tabular}

TGP

$\begin{array}{lllll}\text { Grupo A } & 25 & 26.0 & 25.0 & 24.0 \\ \text { Grupo B } & 14 & 27.0 & 23.0 & 29.0 \\ \text { Total } & 39 & 26.7 & 24.2 & 26.2\end{array}$


TABELA VIII

Valores individuais dos 10 pacientes com alterações de Transaminases no pré e/ou pós-tratamento com oxamniquine em xarope

\begin{tabular}{|c|c|c|c|c|c|c|c|}
\hline \multirow{2}{*}{ Grupo } & \multirow{2}{*}{ N. } & \multicolumn{3}{|c|}{ TGO } & \multicolumn{3}{|c|}{ TGP } \\
\hline & & Pré-trat. & $\begin{array}{l}48 \text { hs } \\
\text { após }\end{array}$ & $\begin{array}{c}10 \text { dias } \\
\text { aposs }\end{array}$ & Pré-trat. & $\begin{array}{l}48 \mathrm{hs} \\
\text { após }\end{array}$ & $\begin{array}{c}10 \text { dias } \\
\text { após }\end{array}$ \\
\hline \multirow[t]{6}{*}{ A } & 11 & 43 & 39 & 28 & 24 & 21 & 22 \\
\hline & 17 & 46 & 27 & 30 & 38 & 52 & 32 \\
\hline & 19 & 33 & 44 & 48 & 48 & 38 & 42 \\
\hline & 20 & 110 & 110 & 60 & 110 & 79 & 58 \\
\hline & 21 & 40 & 54 & 48 & 36 & 36 & 34 \\
\hline & 23 & 26 & 48 & 30 & 22 & 46 & 26 \\
\hline \multirow[t]{4}{*}{ B } & 28 (HE) & 48 & 32 & 40 & 30 & 27 & 60 \\
\hline & 30 & 50 & 26 & 30 & 34 & 18 & 22 \\
\hline & 37 (HE) & 70 & 80 & 28 & 36 & 40 & 18 \\
\hline & 39 & 30 & 28 & 42 & 30 & 22 & 36 \\
\hline
\end{tabular}

\section{Função renal}

Nenhuma alteração urinária foi observada. As dosagens de uréia e creatinina foram normais nas 2 etapas (antes, 48 hs e $10^{\circ}$ dia) e o exame de urina não revelou qualquer modificação digna de registro.

\section{Hemograma}

O estudo hematológico indicou apenas uma moderada ou elevada eosinofilia sangüinea, presente já no exame prévio, porém aumentada no 10 . dia pós-tratamento e certamente relacionada a morte maciça de parasitas e libertação de seus componentes antigênicos ${ }^{4}$. As taxas elevadas dos eosinofilia, no período pré-tratamento, relacionavam-se sem dúvida, ao poliparasistismo intestinal das crianças (S. mansoni, A lumbricoides, N. americanus, etc).

\section{Estudo Eletrocardiográfico}

Somente em 2 casos ( $n$. 33 e 36) foram evidenciadas pequenas modificações do ECG, sob forma de diminuição do intervalo PR no exame das $24 \mathrm{hs,}$ em relação ao exame prétratamento.

\section{Estudo Ele troencefalográfico}

Já vimos anteriormente a presença de alguns para-efeitos da esfera neuro-psíquica. Dos 5 casos com modificação de comportamento (agressividade ou excitação) 3 apresentaram alteração inespecífica do eletroencefalograma no período das 12 hs, com o caráter geral mais de excitação.

Em outro grupo de 4 pacientes, constatou-se, no período de $12 \mathrm{hs}$, a mesma variedade de alterações inespecificas do EEG não evidenciados no pré-tratamento, porém sem serem acompanhadas de exteriorização clínica, a não ser tontura e sonolência, observadas também em crianças sem qualquer anormalidade do traçado.

Por outro lado, em um $3^{\circ}$ grupo de 5 crianças, as alterações discretas de EEG, verificadas no exame prévio, não apresentaram exacerbações no exame das 24 hs após o tratamento (somente em uma criança desse grupo foi realizado o exame das $12 \mathrm{hs,} \mathrm{também} \mathrm{sem}$ modificação).

\section{Cura Parasitológica}

No que diz respeito a avaliação parasitológica, o critério da cura, utilizado por nós, foi 
o seguinte: 1. acompanhamento por exames mensais/até 4 a 6 meses pós-tratamento; 2 . negativação de, no mínimo, 4 exames; 3 . nenhum exame positivo. (Hoffman e Kato)

Pela Tabela IX, verifica-se nítida diferença entre os 2 grupos, com resultados superiores pelo esquema de dose única ( $90 \%$ de cura) em comparação ao de dose fracionada $(50 \%)$. Ressalta-se, porém, a lém do longo espaço entre as 2 tomadas do medicamento (12 hs) o número relativamente pequeno de casos tratados $\mathrm{e}$ acompanhados em ambos os grupos. No total dos pacientes, a cura parasitológica foi de $\mathbf{7 0 \%}$.

TABELA IX

Tratamento da Esquistossomose em crianças, com Oxamniquine-xarope. Cura parasitológica

\begin{tabular}{ccccc}
\hline Sub-grupo & $\begin{array}{c}\text { N. casos } \\
\text { tratados }\end{array}$ & $\begin{array}{c}\text { N. } \\
\text { controlados }\end{array}$ & \multicolumn{2}{c}{ N. casos curados } \\
\hline $\begin{array}{c}\text { Grupo A } \\
(20 \mathrm{mg} \times 1)\end{array}$ & 25 & 21 & 19 & $\%$ \\
\hline $\begin{array}{c}\text { Grupo B } \\
(10 \mathrm{mg} \times 2)\end{array}$ & 15 & 12 & 6 & 50.0 \\
\hline Total & 40 & 33 & 25 & 70.2 \\
\hline
\end{tabular}

Os pacientes não curados foram separados na Tabela $X$. A constataçâo de positividade parasitológica pós-tratamento foi verificada habitualmente entre o $4 .^{\circ}$ e $\circ 60^{\circ}$ mês, após negativar os exames iniciais, todavia, essa positividade, correspondeu quase sempre a uma acentuada queda do número de ovos $\mathrm{p} / \mathrm{gr}$ de fezes, provavelmente correlacionada a uma redução do grau de infecção. Fez exceção o caso n. 27 , de forma hepato-esplênica que apresentou moderada positividade desde o 1 ? mês e permaneceu nos meses anteriores. $\dot{E}$ possível que, em alguns desses zasos, em vez de falha real da terapêutica, tivesse ocorrido reinfecção, em virtude da impossibilidade de controlar inteiramente novos contactos com água poluída em algumas dessas crianças que residiam em zonas endêmicas.

A presença de tontura transitória continua sendo o efeito colateral mais importante determinado pela Oxamniquine, (22.5\%) embora menos acentuada (nenhum grau 3 ) nesse grupo de criança com o uso de xarope. Todavia, outros sintomas de esfera neuro-psíquica estiveram também presentes, em incidência maior do que a verificada nas experiências anteriores, com dose menor, em adultos ${ }^{1,2}$. Queremos nos referir sobretudo a sonolência, por algumas horas $(20 \%)$ e as modificações de comportamento das crianças (12.5\%) quer sob forma de excitação e excesso de euforia, quer sob aspecto de agressividade, ambas permanecendo por todo um dia e raramente se estendendo, menos intensa, ao $2{ }^{\circ}$ dia. Manifestações equivalentes e esporádicas sob forma de sensação de flutuação ${ }^{5}$, desorientação ${ }^{6}$, leve obnublição ${ }^{2}$, discreta alucinação ${ }^{7}$, agressividade ${ }^{7}$ e convulsões ${ }^{6}$ tem sido assinalados raramente na literatura médica. Dado o caráter transitório dessas manifestações, durando apenas algumas horas, é possivel que elas não apareçam ou somente de forma atenuada, caso a droga seja utilizada a noite antes do paciente deitar-se, aspecto a ser melhor investigado.

Que este efeito neuro-psíquico é leve e passageiro, atestam os estudos eletroencefalográficos negativos, realizados em adultos com $24 \mathrm{hs}^{6}$ ou $72 \mathrm{hs}^{1,2}$ após a medicação. Todavia, nos exames realizados mais precocemente (12 hs), como no estudo de Katz ${ }^{8}$ e na presente investigação, pode-se despistar discretas modificações inespecíficas do traçado que não persistem no exarne de 24 ou $72 \mathrm{hs,} \mathrm{em}$ concordância com a transitoriedade das tontu- 
TABELA $X$

OXAMNIQUE - XAROPE EM CRIANÇAS. CASOS NÃO CURADOS

\begin{tabular}{|c|c|c|c|c|c|c|c|c|c|c|c|c|}
\hline \multirow{2}{*}{ N? } & \multirow{2}{*}{ Iniciais } & \multirow{2}{*}{ Idade } & \multirow{2}{*}{ Sexo } & \multirow{2}{*}{ F. Clin. } & \multirow{2}{*}{ Esquema } & \multirow{2}{*}{$\begin{array}{l}\text { NO ovos } \\
\text { pré-trat. }\end{array}$} & \multicolumn{6}{|c|}{ NQovos pós-tratametno } \\
\hline & & & & & & & 19 & 29 & $3 ?$ & $4 !$ & $5 ?$ & $6 ?$ \\
\hline 13 & LMD & 11 & $\mathbf{F}$ & $\mathrm{HI}$ & A & 1.000 & neg. & neg. & neg. & 20 & neg. & 120 \\
\hline 17 & $\mathrm{NCl}$ & 12 & $M$ & $\mathrm{HI}$ & $A$ & 540 & neg. & neg. & neg. & 80 & neg. & neg. \\
\hline 27 & AAS & 11 & M & HE & B & 1.020 & 400 & neg. & neg. & 440 & 80 & 160 \\
\hline 28 & ALS & 13 & $M$ & $\mathrm{HI}$ & B & 760 & neg. & neg. & neg. & neg. & neg. & 80 \\
\hline 30 & WMS & 7 & M & $\mathrm{HI}$ & B & 920 & neg. & neg. & neg. & neg. & 100 & neg. \\
\hline 31 & EMS & 10 & $F$ & $\mathrm{HI}$ & B & 1.080 & 20 & neg. & neg. & 20 & neg. & neg. \\
\hline 34 & JMO & 13 & $M$ & $\mathrm{HI}$ & B & 660 & neg. & neg. & neg. & neg. & 40 & neg. \\
\hline 35 & EGS & 6 & $F$ & $\mathrm{HI}$ & B & 2.340 & neg. & neg. & neg. & neg. & 80 & neg. \\
\hline
\end{tabular}

Obs.: Houve redução acentuada do número de ovos em todos os casos. 
ras e das alterações neuro-psíquicas referidas acima.

O caráter benígno e efêmero dessa ação sobre o sistema nervoso, está também indicado no estudo otoneurológico realizado por Almeida e colabs (6) em 10 pacientes adultos, 24 hs antes, 24 hs após e 7 dias depois do uso da Oxamniquine em cápsulas $(20 \mathrm{mg} / \mathrm{k})$. Nenhuma alteração da parte auditiva foi observada e somente 1 caso mostrou alteração do exame vestibular, 24 hs após o uso da droga, não persistindo no 70 dia. Esse mesmo paciente já apresentava antecedente de labirintopatia.

Que a existência de antecedentes neuropsíquicos ou de alterações prévias de EEG parecem facilitar, até um certo ponto, o aparecimento das citadas manifestações e/ou de modificações eletroencefalográficas após o uso do medicamento, sugerem a nossa experiência em adultos ${ }^{2}$ e a presente investigação em crianças, embora seja difícil, nesse terreno, delimitar o normal e o patológico. Seja como for, a Oxamniquine parece ter uma pequena ação transitória sobre o sistema nervoso e sobre o órgão vestibular, mais evidente com doses maiores ou em individuos predispostos que necessita ser conhecida, a fim de serem utilizados os meios preventivos e corretivos adequados. No entanto, não parece existir uma verdadeira impregnação do sistema nervoso pela droga (ver estudos do EEG) estando ela longe de ser comparada ao Niridazol ${ }^{9}$.

Em relação as duas formas posológicas, 20 $\mathrm{mg} / \mathrm{k}$ dose única (Grupo $\mathrm{A}$ ) e $20 \mathrm{mg} / \mathrm{k}$ dividida em 2 tomadas (Grupo B) há evidentemente, na atual experiência, uma melhor tolerância para o fracionamento da droga: $73 \%$ de ausência de para-efeitos em comparação com $44 \%$ do Grupo A. Quando presentes, esses efeitos colaterais no Grupo B, mostram-se bem menos intensos do que no Grupo $A$, não se observando, em nenhum caso daquele Grupo, as modificações de comportamento referidas para o Grupo de dose única.

Em relação aos testes hepáticos e de função renal, ao hemograma e ao estudo eletrocardiográfico, ambos os Grupos se comportaram de maneira idêntica, isto é, nenhuma ação peculiar foi comprovada (hepatotóxica, cardiológica, etc.) confirmando a nossa experiência, em adultos, com outras apresentações da dro$\mathrm{ga}^{1,2}$.
Infelizmente, porém, esta melhor tolerância para a dose fracionada não parece corresponder, na presente experiência, com a manutenção do efeito esquistossomicida: $(90 \%)$ de cura parasitológica para o Grupo $A$, em relação a $50 \%$ para o Grupo $B$, segundo o critério estricto adotado, embora tenha ocorrido uma acentuada redução do número de ovos eliminados nos pacientes não curados. É possível que este baixo índice de cura no Grupo B lassim como a sua melhor tolerância) esteja na depen. déncia do longo período (12 hs) entre as 2 tomadas, não permitindo, desta forma, uma suficiente concentração sanguinea da droga em virtude de sua metabolização rápida. É recomendável, portanto, que nas ulteriores investigações esse intervalo seja reduzido para 4 a 6 horas, o que possivelmente facilitará a tolerância com a conservação do efeito terapêutico.

A maioria dos pacientes não curados apresentou negativação dos exames nos primeiros meses, com reaparecimento de pequena quantidade de ovos eliminados entre o 40 e o 60 mês. Ocorreria, nesses casos, retorno da oviposição. inibida temporariamente pela droga? Ou a volta de alguns casais de parasitas, não exterminados, ao sistema mesentérico? Não se pode afastar também a possibilidade, em alguns casos, da ocorrência de reinfestação, dadas as múltiplas oportunidades de contágio das crianças de baixo nível sócio-econômico, residindo em zonas rurais ou na periferia das cidades em precárias condições de saneamento, embora recomendações tenham sempre sido feitas no sentido de evitar, tanto quanto possível, a reinfecção.

O único caso que apresentou positividade em vários exames e em quantidade apreciável de ovos, foi um paciente hepato-esplênico (obs. $n$ ? 27). Em nossa experiência anterior com cápsu$\operatorname{las}^{2}$, os jovens (12-18 anos) hepato-esplênicos também se apresentaram como os mais difíceis de cura. Estaria isto relacionado a um grau maior de infestação, conforme sugere o clássico trabalho de Cheever ${ }^{10}$ e os recentes estudos clínico-epidemiológicos ${ }^{1}::^{2}$. Seja como for, pretendemos, para contornar esta aparente resistência, estabelecer, em futuro próximo, novos esquemas posológicos para os hepato-esplênicos, sobretudo os mais jovens. 


\section{SUMMARY}

The authors present the results of the treatment of 40 children (5 to 14 years) with oxamniquine syrup; 25 of them were given a single dose of $20 \mathrm{mg} / \mathrm{kg} \mathrm{b.w}$. and 15 received the same total dose divided at 12 hours intervals. Tolerance was good; $22,5 \%$ had dizziness and $20 \%$, drowsiness; 5 patients of the first group presented psychological disturbances. $70 \%$ of the patients presented parasitological cure 4 to 6 months after treatment; $(90 \%$ em Group $A$ and $5 \%$ in Group B) a marked egg count reduction was observed.

\section{REFERÊNCIAS BIBLIOGRÁFICAS}

1. COUTINHO, A., DOMINGUES, A.L.C. \& BONFIM, J.R. Treatment of Mansoni Schistosomiasis with Oxamniquine. Rev. Inst. Med. trop. São Paulo, 15 (Supl. 1): 15-34, 1973.

2. DOMINGUES, A.L.C. \& COUTINHO, A. Tratamento da esquistossomose mansônica com Oxamniquine oral. Rev. Inst. Med. trop. São Paulo, 17: 164-180, 1975

3. COUTINHO, A. \& DOMINGUES, A.C.L. Tratamento da esquistossomose in testinal e hepática pela Oxamniquine. Comparação vias intramuscular e oral. Memórias del IV Cong. . de la Socied. Latinoamericana de Hepatologia p. 284-297, 1974

4. COUTINHO, A. Clinical-laboratory manifestations due to the death of worms after specific treatment of schistosomiasis. Brasilia Médica 11:69-80, 1975

5. CAMPOS, R. et all. Tratamento da esquistossomose mansônica pela Oxamniquine em região não endêmica. Resumo apresentado no XII Cong. Soc. Bras. Med. Trop. Belém, fev. 1976.

6. ALMEIDA, F.M.R. et all. Avaliação terapêutica da Oxamniquine na esquistossomose mansônica. Separata, 1976.
7. COURA, J.R. Comunicação pessoal, 1975.

8. KATZ, N., apresentação no Simpósio sobre tratamento da esquistossomose mansônica com Oxamniquine p/via oral, Salvador, jul. 1974.

9. COUTINHO, A.,\& BARRETO, F. T. Treatment of hepatoeplenic schistosomiasis mansoni with Niridazole: relationships among liver function, affective dose and side effects: Ann N.Y. Acad. Sci. 160: 612.628, 1969.

10.CHEEVER, A.W. A quantitative posmortem study of schistosomiasis mansoni in man. Am. J. Trop. Med. Hugg. 18: 38-64, 1968.

11. SIONGOK,T.K.A. et all. Morbidity in shistosomiasis mansoni in relation to intensity of infection: study of a community in Machakos, Kenya. Am. J. Trop. Med. Hyg. 25: 273.284, 1976.

12. LEHMAN; J.S. et all. The intensity and effects of infection with Schistosoma mansoni in a rural community in Northeast Brasil. Am J. Trop. Med. Hyg. 25: 285-294, 1976. 